\title{
Interior Solar-Cycle Changes Detected by Helioseismology
}

R. Howe, F. Hill, and R. W. Komm

National Solar Observatory, 950 N. Cherry Avenue, Tucson AZ 85726-6732, USA

J. Christensen-Dalsgaard

Teoretisk Astrofysik Center, Danmarks Grundforskningfond; and Institut for Fysik og Astronomi, Aarhus Universitet, DK-8000 Aarhus C, Denmark

R. Munk Larsen and J. Schou

HEPL Annex A201, Stanford University, Stanford CA 94305-4085, USA

M. J. Thompson

Astronomy Unit, Queen Mary $\&$ Westfield College, London E1 4NS, UK

J. Toomre

JILA, and Dept. of Astrophysical and Planetary Sciences, University of Colorado, Boulder CO 80309-0440, USA

\begin{abstract}
Helioseismic measurements with the MDI instrument aboard SOHO, and complementary measurements from the GONG network, are revealing changes deep within the Sun as the solar cycle progresses. We present results based on recent data from both experiments, including variations in the rotation rate deep inside the convection zone.
\end{abstract}

\section{Introduction}

Helioseismology uses acoustic modes as probes of the structure and rotation of the solar interior. In this work we concentrate on changes in the solar rotation over the five years covered by the combined observations of MDI and GONG. Earlier work by, for example, Libbrecht (1988) established the now well-known solar rotation profile with rotation approximately constant along radial lines within the convection zone, with shear layers near the surface and at the base of the convection zone. More recently this picture has been refined, for example by Schou et al. (1998), and with the continuous coverage provided by the new observations it has been possible to detect temporal changes in the rotation profile, both near the surface, corresponding to the torsional oscillation (Howe et al. 2000a) and, more unexpectedly, at and below the base of the convection 


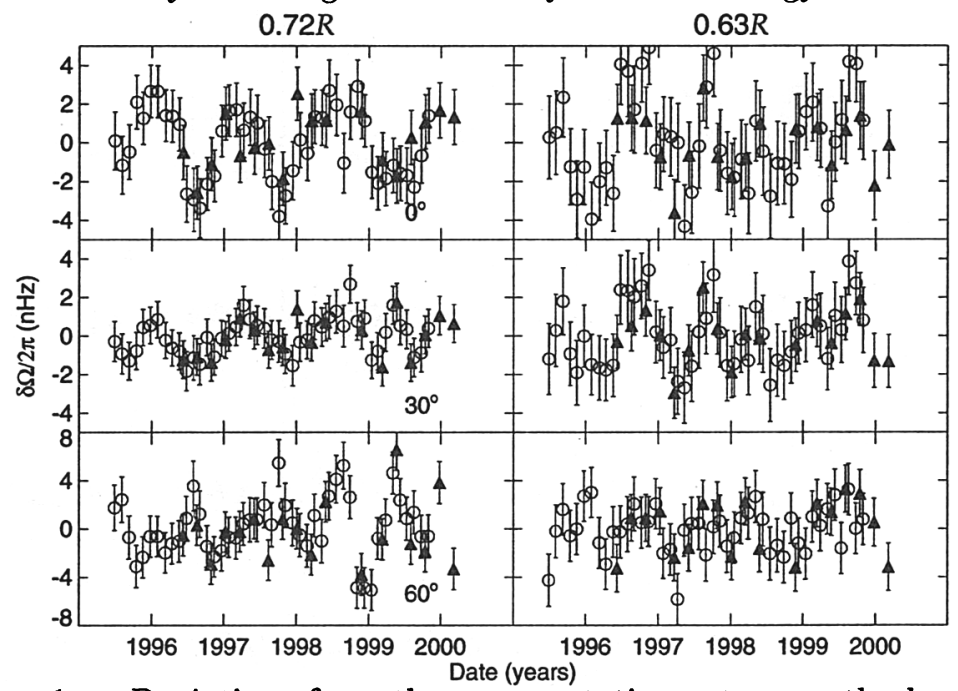

Figure 1. Deviations from the mean rotation rate near the base of the convection zone, at $0,30,60$ degrees latitude, for GONG (open circles) and MDI (filled triangles) RLS data. Left column $-0.72 R_{\odot}$; Right column $-0.63 R_{\odot}$.

zone (Howe et al. 2000b). More up-to-date results on the torsional oscillation can be seen in the paper by Schou (2000).

\section{Data}

We have analysed 45 108-d GONG time series with starting dates 36d apart, each containing approximately 1400 modes up to $l=150$ and covering the period May 1995 to December 1999, and 18 independent 72-d MDI series from May 1996 to April 2000, each with about 3000 modes up to $l=300$. GONG and MDI data were separately analysed to obtain the mode frequencies and rotation coefficients. Each modeset was inverted using two inversion techniques: Regularized Least Squares (RLS) and Optimally Localized Averages (OLA). However, for clarity and brevity we do not show the OLA results here. The mean rotation rate as a function of latitude and radius was then subtracted from each profile to reveal the evolving features. For details, see Howe et al. $(2000 \mathrm{a}, \mathrm{b})$.

\section{Oscillations at the Base of the Convection Zone}

We now have 4 GONG data sets and 2 MDI sets that were not available for the Howe et al. (2000b) paper. As seen in Figure 1, the new data continue the oscillatory trend previously observed near the equator at the base of the convection zone.

In Figure 2, we show the results of a few tests supporting the robustness of the $1.3 \mathrm{y}$ period oscillation at $0.72 R$, latitude $0^{\circ}$ in GONG data. The period is seen in RLS inversions of the coefficients even where only non-overlapping data 

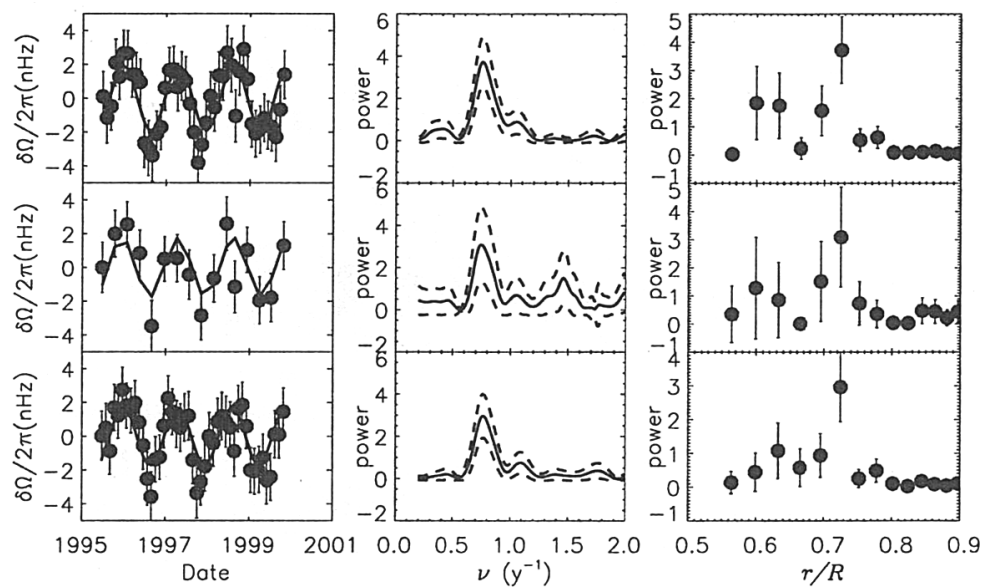

Figure 2. Quantification of the oscillation at $0.72 R_{\odot}, 0^{\circ}:$ Left column shows best sine wave fit to the data, middle column shows 'power spectrum' of best-fit sinewaves, right column shows radial distribution of power in the dominant $0.77 \mathrm{y}^{-1}$ frequency. Top row - GONG RLS data; middle row - GONG RLS data taking only independent points; bottom row - GONG RLS data from individual splittings.

sets are used, and in inversions carried out using individual $m$ splittings rather than coefficients fitted to the frequencies.

Acknowledgments. This work utilizes data obtained by the Global Oscillation Network Group (GONG) project, managed by the National Solar Observatory, which is operated by AURA, Inc. under a cooperative agreement with the National Science Foundation. The data were acquired by instruments operated by the Big Bear Solar Observatory, High Altitude Observatory, Learmonth Solar Observatory, Udaipur Solar Observatory, Instituto de Astrofísico de Canarias, and Cerro Tololo Interamerican Observatory. The Solar Oscillations Investigation (SOI) involving MDI is supported by NASA grant NAG 5-3077 to Stanford University. $\mathrm{SOHO}$ is a mission of international cooperation between ESA and NASA. RWK, and RH in part, were supported by NASA contract S-92698-F. This work was supported in part by the UK Particle Physics and Astronomy Research Council, and by the Danish National Research Foundation through the establishment of the Theoretical Astrophysics Center (TAC).

\section{References}

Howe, R., Christensen-Dalsgaard, J., Hill, F., et al., 2000a, ApJ, 533, L163

Howe, R., Christensen-Dalsgaard, J., Hill, F., et al., 2000b, Science, 287, 2456 Libbrecht, K.G. 1988, in Seismology of the Sun and Sun-like Stars, ed. E.J.Rolfe, ESA, 131

Schou, J., Antia, H.M., Basu, S., et al., 1998, ApJ, 505, 390

Schou, J., 2000, this volume 